\title{
Cecropins as a marker of Spodoptera frugiperda immunosuppression during entomopathogenic bacterial challenge
}

\author{
B. Duvic ${ }^{a, b, *}$, V. Jouana ${ }^{a, b}$, N. Essa ${ }^{a, b}$, P.-A. Girard ${ }^{a, b}$, S. Pagès ${ }^{a, b}$, Z. Abi Khattar ${ }^{a, b}$, N.-A. Volkoff ${ }^{a, b}$, \\ A. Givaudan ${ }^{\mathrm{a}, \mathrm{b}}$, D. Destoumieux-Garzon ${ }^{\mathrm{b}, \mathrm{c}, \mathrm{d}}$, J.-M. Escoubas ${ }^{\mathrm{a}, \mathrm{b}}$
}

a INRA, UMR1333, Laboratoire Diversité, Génomes et Interactions Microorganismes Insectes, Montpellier, France

${ }^{\mathrm{b}}$ Université de Montpellier 2, Place E. Bataillon, 34095 Montpellier cedex 05, France

${ }^{\mathrm{c}}$ CNRS/IFREMER/IRD, UMR5119, Laboratoire Ecologie des Systèmes Marins Côtiers, Montpellier, France

d Université de Montpellier 1, 5 Boulevard Henri IV, 34967 Montpellier cedex 02, France

\author{
*: Corresponding author: B. Duvic, Tel.: +33 467144672 ; fax: +33 467144679 \\ email address : duvic@univ-montp2.fr
}

\section{Abstract :}

An antimicrobial peptide (AMP) of the cecropin family was isolated by HPLC from plasma of the insect pest, Spodoptera frugiperda. Its molecular mass is $3910.9 \mathrm{Da}$ as determined by mass spectrometry. Thanks to the EST database Spodobase, we were able to describe 13 cDNAs encoding six different cecropins which belong to the sub-families $\mathrm{CecA}, \mathrm{CecB}, \mathrm{CecC}$ and $\mathrm{CecD}$. The purified peptide identified as CecB1 was chemically synthesized (syCecB1). It was shown to be active against Gram-positive and Gram-negative bacteria as well as fungi. Two closely related entomopathogenic bacteria, Xenorhabdus nematophila F1 and Xenorhabdus mauleonii VC01 ${ }^{\top}$ showed different susceptibility to syCecB1. Indeed, $X$. nematophila was sensitive to syCecB1 whereas $X$. mauleonii had a minimal inhibitory concentration (MIC) eight times higher. Interestingly, injection of live $X$. nematophila into insects did not induce the expression of AMPs in hemolymph. This effect was not observed when this bacterium was heat-killed before injection. On the opposite, both live and heat-killed $X$. mauleonii induced the expression of AMPs in the hemolymph of $S$. frugiperda. The same phenomenon was observed for another immune-related protein lacking antimicrobial activity. Altogether, our data suggest that Xenorhabdus strains have developed different strategies to supplant the humoral defense mechanisms of $S$. frugiperda, either by increasing their resistance to AMPs or by preventing their expression during such host-pathogen interaction.

\section{Graphical abstract :}
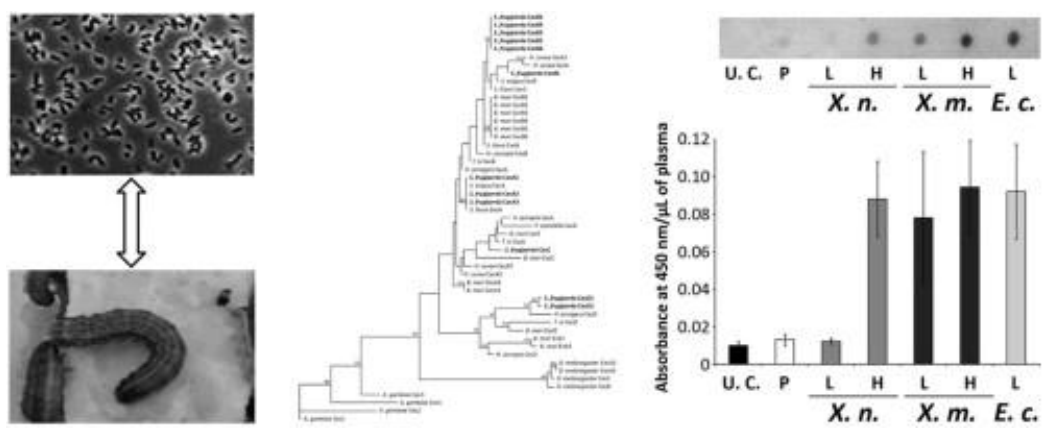

\section{Highlights}

- We first describe the Spodoptera frugiperda cecropin family (SfCec). $>$ SfCec family is composed of 12 members. Two bacteria of Xenorhabdus genera show different susceptibility to SfCec. Xenorhabdus sp. set up different strategies to overcome insect innate immunity.

Keywords : Humoral immunity ; Innate immunity ; Immunosuppression ; Lepidopteran ; Invertebrate 


\section{Introduction}

To fight infection, insects rely on multiple innate defence reactions which include the use of physical barriers together with local and systemic immune responses. These involve phagocytosis and encapsulation by hemocytes (Costa et al., 2005; Russo et al., 1996), proteolytic cascades leading to coagulation (Loof et al., 2011) and melanisation (Kanost et al., 2004), and de novo secretion of antimicrobial peptides (AMPs) (Lemaitre and Hoffmann, 2007). AMPs are widely distributed among living organisms (Garcia-Olmedo et al., 1998; Zasloff, 2002).

Since the first report of an inducible defense reaction in Drosophila (Boman et al., 1972), AMPs have been identified and characterized from the main insect orders Diptera (Kylsten et al., 1990), Lepidoptera (Dickinson et al., 1988; Steiner et al., 1981), Coleoptera (Bulet et al., 1991), Hemiptera (Cociancich et al., 1994) and Hymenoptera (Casteels et al., 1989). On the basis of sequence and structural features, these peptides have been classified into three broad classes: (i) linear peptides with amphipathic $\alpha$-helices, (ii) cysteine-stabilized $\alpha$-helical/ $\beta$-sheet motif containing peptides and (iii) linear peptides with an overrepresentation in proline and/or glycine residues (Bulet and Stocklin, 2005).

Cecropins are among the best known $\alpha$-helical cationic antimicrobial peptides. Mature cecropins are composed of highly basic amino acid residues that can fold into two amphiphatic $\alpha$-helices and integrate into the acidic cell membranes of bacteria leading to their disruption (Steiner et al., 1988). Recent works indicate that cecropins seem to be a target of insect pathogens. Indeed, the bacterialchallenged induction of cecropins from the lepidoptera Plutella xylostella was shown to be inhibited by a symbiotic polyDNA virus (CpBV) of the hymenoptera endoparasitoid Cotesia plutellae (Barandoc et al., 2010). On the other hand, Xenorhabdus nematophila, a Gram-negative bacterium belonging to the Enterobacteriaceae family, kills various insects. These bacteria form a species-specific mutualistic association with the entomopathogenic nematode, Steinernema carpocapsae (Thomas and Poinar, 1979) and are transported and released by nematode vectors into the hemocoel (body cavity) of 
insect hosts. Xenorhabdus initially colonizes the connective tissue surrounding the anterior midgut

52 and hemolymph (bloodstream) of the Lepidoptera Spodoptera littoralis (Sicard et al., 2004), leading

53 to the death of the insect, probably due to a combination of the effects of toxins and septicaemia.

$54 X$. nematophila can independently kill insects and grow within their bodies if introduced into the 55 hemolymph by direct injection (Sicard et al., 2004). X. nematophila proliferates in the hemolymph 56 before the insect dies and must therefore be able to escape the insect immune response. Hence, it 57 was shown that Xenorhabdus inhibits the expression of cecropins (Ji and Kim, 2004). This indicates that different pathogens might use a common strategy in order to establish their pathogenicity.

In this work, we purified a cecropin from the hemolymph of bacterial-challenged larvae of the crop pest, Spodoptera frugiperda. The analysis of Spodobase

(http://bioweb.ensam.inra.fr/spodobase/), a Spodoptera specific cDNA database (Negre et al., 2006), allowed the identification of 13 distinct nucleotide sequences encoding members of the cecropin family. The deduced amino acid sequences led us to classify the $S$. frugiperda cecropins in 4 families (CecA, B, C and D) and to identify the purified cecropin as CecB1. CecB1 was chemically synthesized (syCecB1) and its antimicrobial activity was tested against a panel of microorganisms. Thus, we showed that two closely related entomopathogenic bacteria, Xenorhabdus nematophila F1 strain and Xenorhabdus mauleonii strain $\mathrm{VCO}^{\top}$, were not sensitive to the same extent to syCecB1. Additionally, $X$. nematophila $\mathrm{F} 1$ had an inhibitory effect on cecropin expression whereas $X$. mauleonii $\mathrm{VCO}^{\top}$ did not have such an effect. This difference between the two Xenorhabdus strains could be extended to the global antimicrobial activity present into the hemolymph of infected larvae. Finally, the same results were obtained when we followed the outcome of Spod-11-tox, an immune-related protein lacking antimicrobial activity and mainly expressed by hemocytes (Destoumieux-Garzon et al., 2009). Altogether, these results showed that the two entomopathogenic Xenorhabdus strains use two different strategies in order to circumvent the humoral immune response of their insect host. 


\section{Materials and Methods}

\section{1.1. Insects and Immune Challenge}

Spodoptera frugiperda were reared on artificial diet (Pagès and Ginibre, 2006) at $23^{\circ} \mathrm{C}$ with a photoperiod of $12 \mathrm{~h}$. One day-old sixth-instar larvae were used for the expression studies. Larvae used for antimicrobial peptide purification were bacterial-challenged with Escherichia coli CIP7624 (Gram negative) and Micrococcus luteus CIP5345 (Gram positive)(10 6 bacteria/larva). Eight hours post challenge, hemolymph was collected from a cut abdominal proleg into ice-cold anticoagulant buffer $(69 \mathrm{mM} \mathrm{KCl}, 27$ mM NaCl, 2 mM NaHCO 3,100 mM D-glucose, 30 mM tripotassium citrate, 26

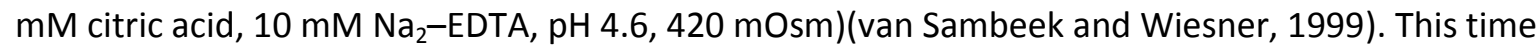
point was chosen because Girard et al. (2008) previously showed that this was the time at which maximum expression of antimicrobial peptides occurred.

\section{Experimental infections were also performed by injection of $10^{6}$ PBS-washed Xenorhabdus} nematophila strain F1 or Xenorhabdus mauleonii strain $\mathrm{VCO}^{\top}$ (from the laboratory collection). The use of this high dose of bacteria was to avoid difference of virulence of the Xenorhabdus sp. In some cases, the bacteria were heat killed by 10 min incubations at $95^{\circ} \mathrm{C}$.

1.2. Plasma (cell-free hemolymph) Purification Procedure as described in (Destoumieux-Garzon et al., 2009)

1.2.1. Extraction and pre-purification by solid-phase extraction (SPE) Hemocytes and plasma were separated by centrifugation at $600 \mathrm{x}$ g during $30 \mathrm{sec}$ at $4^{\circ} \mathrm{C}$. Plasma from bacterial-challenged larvae $(28 \mathrm{~mL})$ was acidified to $\mathrm{pH} 2$ with $1 \mathrm{M} \mathrm{HCl}$. The acidic extraction was performed overnight under gentle shaking at $4^{\circ} \mathrm{C}$. After centrifugation $(16,000 \mathrm{xg}$ for $30 \mathrm{~min}$ at 4 ${ }^{\circ} \mathrm{C}$ ), the supernatant was pre-purified by SPE on Sep-Pak $\mathrm{C}_{18}$ cartridges (Waters) equilibrated with $0.05 \%$ trifluoroacetic acid (TFA). Elutions were performed with $10 \%, 40 \%$ and $80 \%$ acetonitrile in acidified water. All fractions were freeze-dried in a vacuum centrifuge (Speed-Vac, Cryo Rivoire) and subsequently reconstituted with MilliQ water at 1/20 of the initial hemolymph volume. 
1.2.2. HPLC purification

The $40 \%$ Sep-Pak fractions were subjected to reverse phase chromatography on an

UP5NEC25QS column (Interchim) equilibrated in 0.05\% TFA. Separation of the $40 \%$ Sep-Pak fractions was performed with a linear gradient of $0-60 \%$ acetonitrile in acidified water over 80 min at a flow rate of $1 \mathrm{~mL} / \mathrm{min}$.

Antimicrobial activity-containing fractions were purified on the same reversed phase column as above at a controlled temperature of $35^{\circ} \mathrm{C}$. The linear biphasic gradient was composed of $0 \%-28 \%$ acetonitrile in $0.05 \%$ TFA over $5 \mathrm{~min}$, and of $28 \%-45 \%$ over $55 \mathrm{~min}$ at a flow rate of $1 \mathrm{~mL} / \mathrm{min}$.

When needed, the fractions of interest were subjected to a final purification step on a narrowbore reversed phase column (Xbridge BEH130, Waters Associates) at $40^{\circ} \mathrm{C}$ with a flow rate of 0.25 $\mathrm{mL} / \mathrm{min}$ using the biphasic gradients described above.

All HPLC purifications were performed on a Waters HPLC system (Waters 600 pump) equipped with a photodiode array (Waters 996 PDA). Column effluent was monitored by its UV absorption at $225 \mathrm{~nm}$. Fractions corresponding to absorbance peaks were hand collected in polypropylene tubes (Microsorb $75 \mathrm{~mm} \times 12 \mathrm{~mm}$, Nunc immunotubes), concentrated under vacuum (Savant) and reconstituted in MilliQ water (Millipore ${ }^{\mathrm{TM}}$ ) before antimicrobial activity testing.

\subsection{Antimicrobial Assays.}

Antimicrobial activity was assayed against Gram positive bacteria, Gram negative bacteria and 119 fungi (listed in Table 2) based on the liquid growth inhibition assay described previously (Hetru and 120 Bulet, 1997). Poor Broth (PB : $1 \%$ bacto-Tryptone, $0.5 \% \mathrm{NaCl} w / \mathrm{v}, \mathrm{pH} 7.5$ ), and $1 / 2$ Potato Dextrose 121 Broth (Difco) were used for bacterial and fungal growth, respectively. Growth of bacteria was 122 monitored spectrophotometrically at $600 \mathrm{~nm}$ on a multifunctional microplate reader (Tecan infinite 123200 ) while fungal growth was evaluated after 24 and 48 hours at $30^{\circ} \mathrm{C}$ by optical microscopy and 124 measurement of the culture absorbance at $595 \mathrm{~nm}$. MIC values are expressed as the lowest concentration tested that caused $100 \%$ of growth inhibition (micromolar). The bactericidal activity of 
the peptide was assessed by plating cultures. The absence of CFUs on agar plates, after an overnight

127 incubation at $30^{\circ} \mathrm{C}$, was considered indicative of a bactericidal effect.

1.4. Matrix-assisted laser desorption ionization-time of flight (MALDI-TOF) mass spectrometry

Mass spectrum was acquired on Ultraflex (Bruker) in positive mode. The irradiation source was a pulsed nitrogen laser with a wavelength of $337 \mathrm{~nm}$. A solution of $\alpha$-cyano-4-hydroxycinnamic acid $(10 \mathrm{mg} / \mathrm{ml})$ used as matrix and a product solution were mixed in a ratio of $1: 1$. A $1 \mu \mathrm{L}$ aliquot of the matrix/product mixture was deposited and air dried. External mass calibrations were performed with a standard peptide mixture. The analyses were recorded in reflector mode. Mass spectrum was analyzed with Flex Analysis software.

\subsection{Dot-blot assay}

The presence of cecropins in Spodoptera frugiperda plasma from treated or untreated larvae was evaluated by spotting $3 \mu \mathrm{L}$ of $40 \%$ ACN Sep-Pak fraction on a PVDF membrane $(0.22 \mu \mathrm{m}$, ImmunBlot $^{\mathrm{TM}}$, Bio-Rad). Membranes were probed with rabbit anti-Bombyx mori CecB antibodies (Acris Antibodies, Germany) diluted at $300 \mathrm{ng} / \mathrm{mL}$ in PBS containing $0.05 \%$ Tween-20 and $1 \%$ BSA. A horseradish peroxidase coupled anti-rabbit IgG (GE Healthcare) was used at a 1/5,000 dilution for detection by chemiluminescence (ECL Western blotting detection, GE Healthcare) and finally, the PVDF membrane was exposed to Hyperfilm-ECL (GE Healthcare).

\subsection{Enzyme-linked immunosorbent assay (ELISA)}

Spod-11-tox content in the plasma of challenged and unchallenged larvae was measured by ELISA. Eight hours post-challenge, larvae were bled from a cut abdominal proleg into $150 \mu \mathrm{L}$ of icecold anti-coagulant buffer. Tubes were weighed before and after bleeding to measure the volume of hemolymph from each larva. Hemocytes were separated from plasma by centrifugation $(600 \mathrm{x} g$ for $30 \mathrm{sec}$ at $4^{\circ} \mathrm{C}$ ). For ELISA, microtiter plates (Maxisorp Nunc-Immuno Plate ${ }^{\mathrm{TM}}$ ) were coated with plasma ( $50 \mu \mathrm{L} /$ well), and incubated overnight at room temperature, washed three times with $0.05 \%$ Tween- 
15020 in PBS (PBS-T) and blocked by incubation with $0.25 \%$ BSA in PBS-T for $2 \mathrm{~h}$ at room temperature.

1

3

5 6
Anti-Spod-11-tox antibodies (100 ng/mL in PBS-T containing 1\% BSA) were added to each well and the plates were incubated for $1 \mathrm{~h}$ at room temperature. The plates were washed four times with PBS-

T and incubated for $1 \mathrm{~h}$ with peroxidase-linked donkey anti-rabbit IgG (1/5,000 dilution; GE

Healthcare). The plates were washed four times with PBS-T and $100 \mu \mathrm{L}$ of 1-Step ${ }^{\text {TM }}$ Ultra TMB-ELISA

(Pierce) solution was added to each well. Color development was stopped after 30 min by the addition of $100 \mu \mathrm{L}$ of $2 \mathrm{M} \mathrm{H}_{2} \mathrm{SO}_{4}$, and absorbance at $450 \mathrm{~nm}$ was measured with a microplate reader (Tecan Infinite 200).

\subsection{Production of synthetic S. frugiperda CecB1}

Synthetic CecB1 peptide was produced by NeoMPS S.A. (Strasbourg, France) using the t-Boc solid-phase peptide synthesis technology. 


\section{Results}

\subsection{Purification of a Spodoptera frugiperda cecropin}

In previous work, we detected several antimicrobial activities in the plasma (cell-free hemolymph) of bacterial-challenged S. frugiperda larvae (Destoumieux-Garzon et al., 2009). To further characterize the molecules responsible for those activities, we used a classical procedure for purification of antimicrobial peptides (AMPs) (as described in Materials and Methods). Nine fractions were found to contain antimicrobial activity against the three microorganisms tested (Figure 1a). A fraction that eluted around $40 \%$ acetonitrile (indicated by an arrow on Figure 1a) was further purified (Figure 1b). The molecular weight and the $\mathrm{N}$-terminal sequence of the purified molecule were determined by MALDI-TOF-MS (Figure 1c) and Edman degradation (Figure 1d), respectively. Both, the MS data (one single ion at $\mathrm{m} / \mathrm{z}=3,910.9$ ) and the single $\mathrm{N}$-terminal sequence, Arg-Xaa-Lys-Phe-PheLys-Lys-Ile-Glu-Lys-, showed the purity of the collected peptide. These data are consistent with the isolation of a cecropin.

\subsection{Spodoptera frugiperda genome contains genes encoding members of the antimicrobial peptide} cecropin family

In order to further characterize the purified peptide, we analyzed the Spodobase, a Spodoptera 178 ESTs database (http://bioweb.ensam.inra.fr/spodobase/). We identified 189 sequences 179 corresponding to cecropin transcripts. These sequences were clustered by the use of Seqman 180 (Lasergene, DNASTAR inc.) into 13 different contigs whose characteristics are summarized in Table 1. 181 Their lengths ranged from $433 \mathrm{bp}$ up to $992 \mathrm{bp}$ and the number of sequences in each contig varied 182 from 4 up to 31 . On the other hand, the analysis of their nucleotide sequences allowed the 183 identification of five clusters within which contig sequences differed by few nucleotide substitutions 184 likely due to polymorphism as in cluster II (Supplementary Figure 2) or by the presence of gaps 185 mainly in the 3' UTR for clusters I, III and V which might indicate gene duplication (Supplementary 
Figures 1, 3 and 5). However, the alignment of the deduced amino acid sequences (Figure 2)

indicated that the nucleotide sequences encode only 6 different polypeptides composed of 62 or 63 amino acids.

In order to classify the different members of the S. frugiperda cecropin family, deduced amino acid sequences of the mature polypeptides were aligned with a selection of cecropins from two Diptera (Drosophila melanogaster and Anopheles gambiae) and eight Lepidotera (Bombyx mori, Helicoverpa armigera, Hyalophora cecropia, Hyphantria cunea, Plutella xylostella, Spodoptera exigua, Spodotera litura and Trichoplusia ni) (Supplementary Table 1) using Seaview software (Gouy et al., 2010). The alignment was performed by clustalW (Larkin et al., 2007) and the phylogenetic tree (Figure 3) was obtained by Maximum Likelihood method (Guindon et al., 2010). The cladogram shows that all the lepidopteran cecropins cluster apart from the dipteran sequences. The previously described lepidopteran cecropins A, B, C and E cluster together, whereas cecropins D cluster in a separate clade. The S. frugiperda cecropins that are described here for the first time were named (Table 1) according to their positions in the cladogram. The previously described S. litura CecD however appears to be misnamed, clustering with the lepidopteran CecA/B sub-group.

Comparison of the amino acid sequences of $\mathrm{S}$. frugiperda $\mathrm{Cec}$ with the $\mathrm{N}$-terminal sequence obtained from the purified cecropin showed that it corresponded to CecB1 which is the only one that contains, in the mature peptide obtained after removal of the 26-residue signal peptide, a Phe and an lle at positions 4 and 8 , respectively. According to the amino acid sequence deduced from its cDNA, S. frugiperda CecB1 should be a 36-residues peptide with a molecular mass of 3,951.7 Da, 206 while the mass of the purified cecropin is 3,910.9 Da (Figure 1d). This strongly suggests that the 207 mature peptide is generated by an enzymatic cleavage of the carboxy-terminal Gly residue and a C208 terminal amidation as previously reported (Boman et al., 1989), and therefore, has a final molecular 209 mass of 3,894.7 Da. The $16 \mathrm{Da}$ difference found with the mass determined by MALDI-TOF is likely due 210 to an oxidation of the peptide during the mass spectrometry analysis. The oxidation likely occurred 
on the Trp-residue in position 2, which gave no standard PTU-amino acid by Edman degradation (Xaa in Figure 1d).

\subsection{Antimicrobial activity of S. frugiperda CecB1}

To investigate the antimicrobial activity of S. frugiperda CecB1, we used a chemically synthesized CecB1 (syCecB1) produced by the t-Boc solid-phase peptide synthesis technology. syCecB1 is a 35 amino acid peptide corresponding to the mature peptide formed by deletion of the C-terminal Gly and amidated on the Leu at 35 position ( $\left.\mathrm{R}-35-\mathrm{L}-\mathrm{NH}_{2}\right)$. syCecB1 antimicrobial activity was determined against a panel of microorganisms, including Gram-positive and Gram-negative bacteria, and filamentous fungi. The MIC values obtained are reported in Table 2. Like native CecB1, the synthetic peptide was active against M. luteus, E. coli SBS363 and F. oxysporum, the three microorganisms used during the different purification steps of $\mathrm{CecB} 1$. syCecB1 was more active against Gramnegative bacteria than against Gram-positive bacteria as described for cecropins characterized in other insects (Bulet et al., 2003). syCecB1 had bactericidal effects against all the Gram-negative bacteria tested (Table 2).

Interestingly, entomopathogenic microorganisms such as the Gram negative bacterium $S$. marcescens, Gram positive bacteria Bacillus sp., and the two fungi Beauveria bassiana and Nomuraea 227 rileyi, appeared to be less susceptible to syCecB1 than non-pathogenic ones. Finally, X. nematophila $228 \mathrm{~F} 1$ and $X$. mauleonii $\mathrm{VCO}^{\top}$, two closely related entomopathogenic bacteria were not sensitive to the 229 same extent. Indeed, the MIC value for $X$. mauleonii was eight times higher than the one of $X$. 230 nematophila.

231 2.4. Effect of $X$. nematophila $F 1$ and $X$. mauleonii $V \mathrm{CO}^{\top}$ infection on the induction of humoral 232 response in S. frugiperda.

To investigate if the differential sensitivity of the two Xenorhabdus sp. to syCecB1 was 234 correlated to different infection strategies, we evaluated the total Cec plasma content of $S$. 
frugiperda larvae challenged by $X$. nematophila or $X$. mauleonii. Larvae were challenged with PBS or

E. coli (CIP7624) as controls, or with live or heat-killed Xenorhabdus. The $40 \%$ Sep-Pak fractions

(SPE40) (see Materials and Methods) were freeze-dried and reconstituted with MilliQ water at 1/20 of the initial hemolymph volume. Reconstituted SPE40 (3 $\mu$ l) were spotted on nylon membrane which was then probed by commercial antibodies directed towards Bombyx mori cecropins (Figure 4). Immuno-reactivity was observed within the plasma of larvae challenged with heat-killed bacteria of the two strains, $X$. nematophila $\mathrm{F} 1$ and $X$. mauleonii $\mathrm{VCO}^{\top}$ as well as with live $X$. mauleonii $\mathrm{VC}^{\top} 1^{\top}$ and E. coli CIP7624. On the contrary, when insects were challenged with live $X$. nematophila F1, the level of immune-staining was similar to that found in unchallenged larvae. This indicates that larvae challenged with live $X$. nematophila F1 do not induce cecropins.

The above plasma samples were also used in liquid growth inhibition assays using E. coli SBS363 as bacterial target (Table 3). Results show that plasmas of larvae challenged with either live or heatkilled $X$. mauleonii $\mathrm{VCO}^{\top}$ displayed levels of total antimicrobial activity similar to that found in plasma from $E$. coli-challenged larvae. In larvae challenged with live $X$. nematophila F1, total plasma antimicrobial activity was only about $10 \%$ of that seen in insects challenged with $X$. mauleonii or $E$. coli. However, when $X$. nematophila bacteria were heat-killed, the antimicrobial activity reached a level comparable to that present in plasma of $E$. coli-challenged larvae. Again, these results suggest that larvae challenged with live $X$. nematophila F1 have a reduced expression of antimicrobial peptides. Finally, we quantified the production of Spod-11-tox, an immune-related protein lacking antimicrobial activity (Destoumieux-Garzon et al., 2009), in the plasma of larvae challenged by the two Xenorhabdus sp. (Figure 5). The plasma content of Spod-11-tox in E. coli-challenged larvae was 9 times higher than in unchallenged or PBS-injected larvae. Similar high levels of Spod-11-tox were measured in plasma of $X$. mauleonii $\mathrm{VCO}^{\top}$-challenged larvae whether the bacteria were alive or 258 dead. Conversely, Spod-11-tox plasma content of larvae injected with live $X$. nematophila F1 was 259 comparable to that measured in plasma of unchallenged or PBS-challenged larvae whereas in larvae 
260 challenged by heat-killed $X$. nematophila, Spod-11-tox plasma content was similar to that measured

1 in plasma of E. coli-challenged larvae.

Altogether, these results show that while $X$. mauleonii $\mathrm{VCO}^{\top}$ elicits a regular humoral response for insects challenged by bacteria, $X$. nematophila F1 prevents the expression of plasma-soluble immune-related peptides and proteins.

\subsection{X. nematophila secretes an inhibitor of S. frugiperda humoral response.}

To test whether $X$. nematophila was able to prevent humoral immune response, E. coli bacteria 267 were injected alone or with a culture supernatant of $X$. nematophila (Table 4). The antimicrobial 268 activity measured in the hemolymph of such challenged insects was comparable to the one 269 measured in the hemolymph of insects challenged with PBS or live X. nematophila. In other words, 270 the presence of $X$. nematophila culture supernatant was able to inhibit the AMPs expression 271 observed when E. coli was injected alone. Altogether, these results suggest that the reduced 272 expression of antimicrobial activity observed above is due to a bacterial factor produced and 273 secreted by $X$. nematophila. 


\section{Discussion}

In this study, we have identified a family of cecropins (Cec) encoded by sequences present in Spodobase, a Spodoptera frugiperda specific EST library (Negre et al., 2006). This family appears to be composed of 13 members, $3 \mathrm{Cec} A, 7 \mathrm{CecB}$, one $\mathrm{Cec} C$ and $2 \mathrm{CecD}$ according to their cDNA sequences. Recently, 13 cecropin genes were found in the genome of Bombyx mori (Cheng et al., 2006; Tanaka et al., 2008), the only Lepidopteran genome available to date (The International Silkworm Genome Consortium, 2008). However, the 2 CecA and the 6 CecB silkworm cDNA encode only two amino acid sequences (Tanaka et al., 2008). In our work, we show that a similar situation is present in S. frugiperda, since the 3 CecA give only one protein as do 5 of the CecB cDNA. Therefore, we may suggest that Lepidopteran insects have a high degree of cecropin gene duplication.

Moreover, since the database does not represent the whole $S$. frugiperda transcriptome, we cannot rule out the possibility that $S$. frugiperda genome might contain more cecropin genes. The accessibility of $S$. frugiperda genome would help to check this hypothesis.

S. frugiperda Cec are 62 or 63 amino acids long in their prepro-forms which, after posttranslational modifications, generate mature polypeptides of 36,37 and 42 amino acids that possess one feature that is characteristic of most insect cecropins which is the presence of a tryptophan residue in the first or second position. A TMHMM analysis

(http://www.cbs.dtu.dk/services/TMHMM/) indicates that, as already described for other insect cecropins (Bulet and Stocklin, 2005), all S. frugiperda Cec have a long N-terminal, basic and amphipathic $\alpha$-helix (residues 2 to 22 in the mature peptide, positions determined from CecA1 in Figure 2) and a shorter and more hydrophobic C-terminal helix (residues 25 to 34), linked by a highly conserved $\mathrm{Gly}^{23}$-Pro $^{24}$ hinge region.

A phylogenetic analysis conducted with 51 cecropins from a selection of Dipteran and Lepidopteran allowed the classification of Cec from S. frugiperda as members of the cecropin peptide families $\mathrm{Cec} A, \mathrm{CecB}, \mathrm{Cec} C$ and $\mathrm{Cec} D$. Lepidopteran cecropins were found distributed into two main clades, one containing all types of cecropins except CecD. Lepidopteran CecD present several specific 
301 features such as one highly conserved acidic amino acid, Glu ${ }^{6}$ or Asp ${ }^{6}$, and a $\operatorname{Ser}^{20}$ (positions

determined from the CecD1 mature polypeptide in Figure 2). In addition, the highly conserved Ala-

Pro-Glu-Pro sequence which precedes the mature peptide and corresponds to a pro-peptide removed in two steps by a dipeptidyl aminopeptidase in other cecropins (Boman et al., 1989), is absent from CecD sequences. This suggests that they are produced as mature active peptide directly after digestion by a signal peptidase at the carboxy-terminus of $\mathrm{Ala}^{-1}$. Finally, although this phylogenetic tree does not give a lot of information on the evolutionary history of the cecropin gene, it may indicate that some insects Cec have likely been wrongly named such as for example S. litura CecD that we propose to rename S. litura CecB according to its position in the cladogram.

The $\alpha$-helical linear antimicrobial peptides of insects, such as cecropins, are mostly active against bacteria, with a higher efficacy on Gram negative than Gram positive strains (Choi et al., 2000; Kylsten et al., 1990; Samakovlis et al., 1990). They have also been shown to be active against some fungi (Ekengren and Hultmark, 1999). As expected, syCecB1 had a similar profile of antimicrobial activity. It is noteworthy that the two strains of entomopathogenic fungi tested here were not susceptible to syCecB1 at concentrations as high as $25 \mu \mathrm{M}$. By contrast, entomopathogenic Xenorhabdus nematophila strain F1 was as sensitive as the most susceptible strains, Escherichia coli CIP7624 and Micrococcus luteus. In contrast, $X$. mauleonii $\mathrm{VCO}^{\top}$, an insect pathogen closely related 318 to $X$. nematophila, was much less sensitive to syCecB1. We therefore studied the effect of these two 319 bacteria on the cecropins expression in S. frugiperda. Our results showed that $X$. nematophila F1 was 320 able to inhibit the expression of cecropins in S. frugiperda. This is reminiscent of two recent studies 321 showing that microorganisms such as a polyDNA bracovirus from Cotesia plutellae or $X$. nematophila 322 inhibited the expression of cecropins in Plutella xylostella (Barandoc et al., 2010) and Spodoptera 323 exigua (Ji and Kim, 2004), respectively. Moreover, X. nematophila had also an inhibitory activity on 324 the expression of Spod-11-tox, an immune-related protein inducible by infection (Girard et al., 2008). 325 Because only live $X$. nematophila controls the expression of host immune-related genes, it is likely 326 that this occurs through the secretion by the bacteria of immunosuppressive products that prevent 
327 the host immune response. This hypothesis is reinforced by the fact that the injection of supernatant

from $X$. nematophila culture prevents the induction of antimicrobial activity by $E$. coli. It is known that $X$. nematophila secretes some inhibitory metabolites against insect immune-associated phospholipase $A_{2}\left(P L A_{2}\right)$ to suppress both the cellular and humoral immune responses in the hemocoel of target insects (Kim et al., 2005; Song et al., 2011). However, Ji and Kim (Ji and Kim, 2004) demonstrated that the use of specific $\mathrm{PLA}_{2}$ inhibitors did not inhibit antimicrobial activity or cecropin gene expression when Spodoptera were infected with heat-killed $X$. nematophila suggesting that the inhibition of $X$. nematophila on the expression of the antimicrobial peptide is not related to the inhibition of the eicosanoid pathway. Consequently, the $X$. nematophila-induced inhibition of two different immune-related genes allows us to raise the hypothesis that the bacterial virulence factor may interact with an insect molecular target upstream of the genes, cecropins and Spod-11-tox. Over the past years, several works have been performed to study the interactions between insect pathogens and different defense mechanisms of insects. Hence, it was shown in Drosophila melanogaster that during the early steps of infection, the highly virulent Pseudomonas aeruginosa PA14 strain down-regulates not only cecropins but also IMD pathway-dependent AMPs such as attacins and defensin (Apidianakis et al., 2005). We are currently involved in the characterization of the immunosuppressive factor present in supernatant from $X$. nematophila culture.

Another major result from this study is that two closely related pathogens of insects have 345 evolved divergent strategies to overcome the host defense reactions. Indeed, while $X$. nematophila 346 evades the host antimicrobial response by repressing the expression of AMPs, the other pathogen, $X$. 347 mauleonii strain $\mathrm{VCO}^{\top}$, induces cecropin expression but is resistant to high concentration of SfCecB1. 348 Therefore, these two species of entomopathogenic bacteria use different strategies to evade the 349 host antimicrobial response either by developing AMP resistance mechanisms or by preventing AMP 350 expression. 


\section{Acknowledgements}

The authors wish to thank Clotilde Gibard, Gaëtan Clabots and Mathieu Jambart for rearing Spodoptera frugiperda and Jean-Claude Ogier for helpful discussions on phylogenetic analysis. This work was supported by grants from the French Institut National de la Recherche Agronomique. JME is funded by the Centre National de la Recherche Scientifique.

Nucleotide sequences reported in this paper have been submitted to the GenbankTM/EBI Data 358 Bank with the following accession numbers DY773722, DY773924, DY776416, DY774879, DY778990, 359 DY774910, DY775152, DY779994, DY775699, DY774316 and DY777111 for Sf1F00836, Sf1F01169, 360 Sf1F04795, Sf1F02507, Sf1F09170, Sf1F02553, Sf1F02902, Sf1F11023, Sf1F03731, Sf1F01749 and 361 Sf1F05978 


\section{Figure legends}

Figure 1: Cecropin purification from fractionated S. frugiperda plasma.

(a) a $40 \%$ Sep-Pak fraction from the extraction of immunized S. frugiperda plasma was subjected to reversed phase HPLC on a UPNEC25QS column (Interchim) using a 0-60\% linear gradient (dashed line) of acidified acetonitrile over $80 \mathrm{~min}$. Abs, Absorbance monitored at $225 \mathrm{~nm}$. Antimicrobial activity against Escherichia coli SBS363 (black rectangles), Micrococcus luteus CIP5345 (grey rectangles) and Fusarium oxysporum (white rectangles) was measured by liquid growth inhibition assays. (b) Final purification step with a Xbridge BEH130 narrowbore column with a $0-28-45 \%$ biphasic gradient of acidified acetonitrile over 5 and $40 \mathrm{~min}$. (c) The molecular mass of the purified peptide was measured by MALDI-TOF MS (Ultraflex). (d) The N-terminal sequence of the purified peptide was determined by Edman degradation.

Figure 2: Alignment of deduced amino acid sequences of mature cecropins from Spodoptera frugiperda. Sequences were analysed by ClustalW. Arrow head indicates maturation cleavage site. Asterisks indicate differences between CecD1 (Sf1F01749) and CecD2 (Sf1F05978). Identical residues are indicated by Dots.

Figure 3: Phylogenetic analysis of S. frugiperda cecropins. 39 amino acid sequences of mature cecropins from two Dipteran (Drosophila melanogaster and Anopheles gambiae) and eight Lepidopteran (Bombyx mori, Helicoverpa armigera, Hyalophora cecropia, Hyphantria cunea, Plutella xylostella, Spodoptera exigua, Spodotera litura and Trichoplusia ni) were aligned together with Spodoptera frugiperda cecropins using ClustalW and the phylogenetic tree was obtained by the Maximum Likelyhood method. Bootstrap values of 1000 trials are indicated as numbers. Sequences used in this analysis are listed in Supplementary Table 1.

Figure 4: Effect of Xenorhabdus nematophila F1 and Xenorhabdus mauleonii $\mathrm{VCO}^{\top}$ on the expression of Spodoptera frugiperda cecropins. Fractionated plasma samples (40\% ACN eluted fraction after 
SEP) of S. frugiperda after challenges as indicated (at least 20 larvae per challenge condition) were spotted on nylon membrane. Then, immuno-blot was stained with anti-Bombyx mori CecB antibodies (Acris Antibodies, Germany). U.C.: unchallenged, P: PBS-challenged, L: live, H: heat-killed, X. $n$. : Xenorhabdus nematophila F1, X. m.: Xenorhabdus mauleonii VC01 ${ }^{\top}$, E. c.: Escherichia coli CIP7624. Samples used were: reconstituted plasmas (RP) or plasmas diluted 1:1 or 1:10 in PBS as indicated to the left.

Figure 5: Effect of Xenorhabdus nematophila F1 and Xenorhabdus mauleonii VC01 ${ }^{\top}$ on Spod-11-tox plasma content. Spod-11-tox content in S. frugiperda plasmas was measured by ELISA as described in material and methods section. For each experimental condition, Spod-11-tox content was individually measured into the plasma of at least 20 larvae. Values represent the mean of three independent experiments while error bars show standard deviation. ${ }^{*} p<0.001$ (as determined by Student t-test) compared to PBS injected larvae. U.C.: unchallenged, P: PBS-challenged, L: live, H: heat-killed, $X$. n.: Xenorhabdus nematophila F1, X. m.: Xenorhabdus mauleonii VC01 ${ }^{\top}$, E. c.: Escherichia coli CIP7624.

Supplementary Figures 1 to 5: Cecropin-encoding contigs (see Table 1) present in the Spodobase (Negre et al., 2006) were aligned using Clustal W. Identical nucleotides in Clusters I, II and V are boxed. In the case of Cluster III, boxes indicate residues which are identical in, at least, 3 sequences. ORFs are indicated by the black line. 


\section{References}

Apidianakis, Y., Mindrinos, M.N., Xiao, W., Lau, G.W., Baldini, R.L., Davis, R.W., Rahme, L.G., 2005. Profiling early infection responses: Pseudomonas aeruginosa eludes host defenses by suppressing antimicrobial peptide gene expression. Proceedings of the National Academy of Sciences of the United States of America 102, 2573-2578.

Barandoc, K.P., Kim, J., Kim, Y., 2010. Cotesia plutellae bracovirus suppresses expression of an antimicrobial peptide, cecropin, in the diamondback moth, Plutella xylostella, challenged by bacteria. Journal of Microbiology 48, 117-123.

Boman, H.G., Boman, I.A., Andreu, D., Li, Z.Q., Merrifield, R.B., Schlenstedt, G., Zimmermann, R., 1989. Chemical Synthesis and Enzymic Processing of Precursor Forms of Cecropins A and B. Journal of Biological Chemistry 264, 5852-5860.

Boman, H.G., Nilsson, I., Rasmuson, B., 1972. Inducible antibacterial defence system in Drosophila. Nature 237, 232-235.

Bulet, P., Charlet, M., Hetru, C., 2003. Innate Immunity, in: Ezekowitz, R.A.B., Hoffmann, J.A. (Eds.), Infectious Disease. Humana Press, Totowa, NJ, pp. 89-107.

Bulet, P., Cociancich, S., Dimarcq, J.L., Lambert, J., Reichhart, J.M., Hoffmann, D., Hetru, C., Hoffmann, J.A., 1991. Insect immunity. Isolation from a coleopteran insect of a novel inducible antibacterial peptide and of new members of the insect defensin family. Journal of Biological Chemistry 266, 24520-24525.

Bulet, P., Stocklin, R., 2005. Insect antimicrobial peptides: structures, properties and gene regulation. Protein and Peptide Letters 12, 3-11.

Casteels, P., Ampe, C., Jacobs, F., Vaeck, M., Tempst, P., 1989. Apidaecins: antibacterial peptides from honeybees. EMBO Journal 8, 2387-2391.

Cheng, T., Zhao, P., Liu, C., Xu, P., Gao, Z., Xia, Q., Xiang, Z., 2006. Structures, regulatory regions, and inductive expression patterns of antimicrobial peptide genes in the silkworm Bombyx mori. Genomics 87, 356-365.

Choi, C.S., Lee, I.H., Kim, E., Kim, S.I., Kim, H.R., 2000. Antibacterial properties and partial cDNA sequences of cecropin-like antibacterial peptides from the common cutworm, Spodoptera litura. Comparative Biochemistry and Physiology 125C, 287-297.

Cociancich, S., Dupont, A., Hegy, G., Lanot, R., Holder, F., Hetru, C., Hoffmann, J.A., Bulet, P., 1994. Novel inducible antibacterial peptides from a hemipteran insect, the sap-sucking bug Pyrrhocoris apterus. Biochemical Journal 300 ( Pt 2), 567-575.

Costa, S.C., Ribeiro, C., Girard, P.A., Zumbihl, R., Brehelin, M., 2005. Modes of phagocytosis of Grampositive and Gram-negative bacteria by Spodoptera littoralis granular haemocytes. Journal of Insect Physiology 51, 39-46.

Destoumieux-Garzon, D., Brehelin, M., Bulet, P., Boublik, Y., Girard, P.A., Baghdiguian, S., Zumbihl, R., Escoubas, J.M., 2009. Spodoptera frugiperda X-tox protein, an immune related defensin rosary, has lost the function of ancestral defensins. PLoS One 4, e6795.

Dickinson, L., Russell, V., Dunn, P.E., 1988. A family of bacteria-regulated, cecropin D-like peptides from Manduca sexta. Journal of Biological Chemistry 263, 19424-19429.

Ekengren, S., Hultmark, D., 1999. Drosophila cecropin as an antifungal agent. Insect Biochemistry and Molecular Biology 29, 965-972.

Garcia-Olmedo, F., Molina, A., Alamillo, J.M., Rodriguez-Palenzuela, P., 1998. Plant defense peptides. Biopolymers 47, 479-491.

Girard, P.A., Boublik, Y., Wheat, C.W., Volkoff, A.N., Cousserans, F., Brehelin, M., Escoubas, J.M., 2008. $X$-tox: an atypical defensin derived family of immune-related proteins specific to Lepidoptera. Developmental and Comparative Immunology 32, 575-584.

Gouy, M., Guindon, S., Gascuel, O., 2010. SeaView version 4: A multiplatform graphical user interface for sequence alignment and phylogenetic tree building. Molecular Biology and Evolution 27, 221-224. 
Guindon, S., Dufayard, J.F., Lefort, V., Anisimova, M., Hordijk, W., Gascuel, O., 2010. New algorithms and methods to estimate maximum-likelihood phylogenies: assessing the performance of PhyML 3.0 Systematic Biology 59, 307-321.

Hetru, C., Bulet, P., 1997. Strategies for the isolation and characterization of antimicrobial peptides of invertebrates. Methods in Molecular Biology 78, 35-49.

Ji, D., Kim, Y., 2004. An entomopathogenic bacterium, Xenorhabdus nematophila, inhibits the expression of an antibacterial peptide, cecropin, of the beet armyworm, Spodoptera exigua. Journal of Insect Physiology 50, 489-496.

Kanost, M.R., Jiang, H., Yu, X.Q., 2004. Innate immune responses of a lepidopteran insect, Manduca sexta. Immunological Reviews 198, 97-105.

Kim, Y., Ji, D., Cho, S., Park, Y., 2005. Two groups of entomopathogenic bacteria, Photorhabdus and Xenorhabdus, share an inhibitory action against phospholipase A2 to induce host immunodepression. Journal of Invertebrate Pathology 89, 258-264.

Kylsten, P., Samakovlis, C., Hultmark, D., 1990. The cecropin locus in Drosophila; a compact gene cluster involved in the response to infection. EMBO Journal 9, 217-224.

Larkin, M.A., Blackshields, G., Brown, N.P., Chenna, R., McGettigan, P.A., McWilliam, H., Valentin, F., Wallace, I.M., Wilm, A., Lopez, R., Thompson, J.D., Gibson, T.J., Higgins, D.G., 2007. Clustal W and Clustal X version 2.0. Bioinformatics 23, 2947-2948.

Lemaitre, B., Hoffmann, J., 2007. The host defense of Drosophila melanogaster. Annual Review of Immunology 25, 697-743.

Loof, T.G., Schmidt, O., Herwald, H., Theopold, U., 2011. Coagulation systems of invertebrates and vertebrates and their roles in innate immunity: the same side of two coins? Journal of Innate Immunity 3, 34-40.

Negre, V., Hotelier, T., Volkoff, A.N., Gimenez, S., Cousserans, F., Mita, K., Sabau, X., Rocher, J., LopezFerber, M., d'Alencon, E., Audant, P., Sabourault, C., Bidegainberry, V., Hilliou, F., Fournier, P., 2006. SPODOBASE: an EST database for the lepidopteran crop pest Spodoptera. BMC Bioinformatics 7, 322. Pagès, S., Ginibre, N., 2006. Evaluation de la virulence de bactéries entomopathogènes: Mise en place au laboratoire d'un bio essai reproductible en conditions contrôlées. Les Cahiers Techniques de I'I.N.R.A. 57, 11-19.

Russo, J., Dupas, S., Frey, F., Carton, Y., Brehelin, M., 1996. Insect immunity: early events in the encapsulation process of parasitoid (Leptopilina boulardi) eggs in resistant and susceptible strains of Drosophila. Parasitology 112 ( Pt 1), 135-142.

Samakovlis, C., Kimbrell, D.A., Kylsten, P., Engstrom, A., Hultmark, D., 1990. The immune response in Drosophila: pattern of cecropin expression and biological activity. The EMBO journal 9, 2969-2976. Sicard, M., Brugirard-Ricaud, K., Pages, S., Lanois, A., Boemare, N.E., Brehelin, M., Givaudan, A., 2004. Stages of infection during the tripartite interaction between Xenorhabdus nematophila, its nematode vector, and insect hosts. Applied and Environmental Microbiology 70, 6473-6480.

Song, C., Seo, S., Shrestha, S., Kim, Y., 2011. Bacterial Metabolites of an Entomopathogenic Bacterium, Xenorhabdus nematophila, Inhibit a Catalytic Activity of Phenoloxidase of the Diamondback Moth, Plutella xylostella. Journal of Microbiology and Biotechnology 21, 317-322.

Steiner, H., Andreu, D., Merrifield, R.B., 1988. Binding and action of cecropin and cecropin analogues: antibacterial peptides from insects. Biochimica et Biophysica Acta 939, 260-266.

Steiner, H., Hultmark, D., Engstrom, A., Bennich, H., Boman, H.G., 1981. Sequence and specificity of two antibacterial proteins involved in insect immunity. Nature 292, 246-248.

Tanaka, H., Ishibashi, J., Fujita, K., Nakajima, Y., Sagisaka, A., Tomimoto, K., Suzuki, N., Yoshiyama, M., Kaneko, Y., Iwasaki, T., Sunagawa, T., Yamaji, K., Asaoka, A., Mita, K., Yamakawa, M., 2008. A genome-wide analysis of genes and gene families involved in innate immunity of Bombyx mori. Insect Biochem Mol Biol 38, 1087-1110.

The International Silkworm Genome Consortium, 2008. The genome of a lepidopteran model insect, the silkworm Bombyx mori. Insect Biochemistry and Molecular Biology 38, 1036-1045. 
Thomas, G.M., Poinar, G.O., Jr., 1979. Xenorhabdus gen. nov., a genus of entomopathogenic nematophilic bacteria of the family Enterobacteriaceae. International Journal of Systematic Bacteriology 29, 352-360.

van Sambeek, J., Wiesner, A., 1999. Successful parasitation of locusts by entomopathogenic nematodes is correlated with inhibition of insect phagocytes. Journal of Invertebrate Pathology 73, 154-161.

Zasloff, M., 2002. Antimicrobial peptides of multicellular organisms. Nature 415, 389-395. 
Table 1: Analysis of the SpodoBase, a Spodoptera EST database (Negre et al., 2006). This database is composed of cDNA sequences from three different tissues (hemocytes, fat body and midgut) as well as the cell line Sf9.

\begin{tabular}{|c|c|c|c|c|c|c|c|}
\hline Contig & Name & Nbr of EST & Length & Best BLAST & E_value & Score & $\begin{array}{c}\text { Deduced peptide } \\
\text { (AA) }\end{array}$ \\
\hline Sf1F00836 & CecA1 & 14 & 498 & AF142341.1 Antimicrobial protein cecropin A [S. litura] & $4 e-31$ & 117 & 62 \\
\hline Sf1F01169 & CecA2 & 16 & 494 & AF142341.1 Antimicrobial protein cecropin A [S. litura] & $4 e-36$ & 117 & 62 \\
\hline Sf1F04795 & CecA3 & 31 & 505 & $\underline{\text { AF142341.1 Antimicrobial protein cecropin A [S. litura] }}$ & $1 e-31$ & 117 & 62 \\
\hline Sf1F02507 & CecB1 & 25 & 471 & GU182910.1 Antimicrobial protein cecropin $3[$ H. armigera] & $4 e-21$ & 108 & 62 \\
\hline Sf1F09170 & CecB1 & 4 & 436 & GU182910.1 Antimicrobial protein cecropin $3[$ H. armigera] & $4 e-21$ & 108 & 62 \\
\hline Sf1F02553-1 & CecB4 & 10 & 598 & AF142342.1 Antibacterial protein cecropin B [S. litura] & $2 \mathrm{e}-17$ & 97 & 63 \\
\hline Sf1F02553-2 & CecB2 & 9 & 992 & $\underline{\text { AF142342.1 Antibacterial protein cecropin B [S. litura] }}$ & $2 \mathrm{e}-19$ & 94 & 63 \\
\hline Sf1F02902 & CecB3 & 10 & 915 & AF142342.1 Antibacterial protein cecropin B [S. litura] & $3 e-18$ & 96 & 63 \\
\hline Sf1F11023-1 & CecB5 & 12 & 804 & $\underline{\text { AF142342.1 Antibacterial protein cecropin B [S. litura] }}$ & $3 e-25$ & 123 & 63 \\
\hline Sf1F11023-2 & CecB6 & 10 & 858 & $\underline{\text { AF142342.1 Antibacterial protein cecropin B [S. litura] }}$ & $1 \mathrm{e}-15$ & 93 & 63 \\
\hline Sf1F03731 & $\mathrm{CecC}$ & 14 & 433 & GU182909.1 Antibacterial peptide cecropin 2 [H. armigera] & $2 e-18$ & 97 & 63 \\
\hline Sf1F01749 & CecD1 & 15 & 505 & EU041763.1 Antimicrobial peptide CecD [H. armigera] & $6 e-25$ & 121 & 63 \\
\hline Sf1F05978 & CecD2 & 19 & 498 & EU041763.1 Antimicrobial peptide CecD [H. armigera] & $8 e-24$ & 117 & 63 \\
\hline
\end{tabular}

Clusters and deduced amino acid sequences were determined using SeqMan and EditSeq (Software suite, Lasergene, DNASTAR inc.), respectively. tblastx were performed at NCBI (http://blast.ncbi.nlm.nih.gov/Blast.cgi). 
Table 2: Activity of synthetic Cecropin B1 on various micro-organisms.

\begin{tabular}{|c|c|c|}
\hline Micro-organisms & $\mathrm{MIC}(\mu \mathrm{M})$ & Activity \\
\hline \multicolumn{3}{|l|}{ Gram positive bacteria } \\
\hline Bacillus cereus CIP14579* & 20 & Bacteriostatic \\
\hline Bacillus thuringiensis* & 20 & Bactericidal \\
\hline Micrococcus luteus CIP5345 & 1.25 & Bactericidal \\
\hline Staphylococcus aureus CIP103428 & 20 & Bacteriostatic \\
\hline \multicolumn{3}{|l|}{ Gram negative bacteria } \\
\hline Escherichia coli CIP7624 & 1.25 & Bactericidal \\
\hline Escherichia coli SBS363 & 0.08 & Bactericidal \\
\hline Klebsiella pneumoniae 100633 & 10 & Bactericidal \\
\hline Salmonella enterica CIP5858 & 10 & Bactericidal \\
\hline Serratia marcescens $363^{*}$ & $>20$ & n. d. \\
\hline X. mauleonii $\mathrm{VCO}^{\top *}{ }^{*}$ & 10 & Bactericidal \\
\hline X. nematophila $\mathrm{F}^{*}{ }^{*}$ & 1.25 & Bactericidal \\
\hline \multicolumn{3}{|l|}{ Fungi and Yeast } \\
\hline Fusarium oxysporum & 2.5 & Fungicidal \\
\hline Beauveria bassiana* & $>25$ & n. d. \\
\hline Nomuraea rileyi* & $>25$ & n. d. \\
\hline
\end{tabular}

ॠsed during the different purification steps.

* Entomopathogenic microorganisms.

n. d., not determined 
Table 3: Effect of Xenorhabdus nematophila $\mathrm{F} 1$ and Xenorhabdus mauleonii $\mathrm{VCO}^{\top}$ on the expression of Spodoptera frugiperda antimicrobial peptides directed towards Escherichia coli.

\begin{tabular}{|c|c|c|c|c|c|c|c|}
\hline \multirow{3}{*}{ Experiments } & \multicolumn{7}{|c|}{ Larval treatment } \\
\hline & \multirow{2}{*}{ U.C. } & \multirow{2}{*}{ PBS } & \multirow{2}{*}{ E. coli } & \multicolumn{2}{|c|}{ X. nematophila } & \multicolumn{2}{|c|}{ X. mauleonii } \\
\hline & & & & Live & Heat-killed & Live & Heat-killed \\
\hline$N^{\circ} 1$ & $1 / 4$ & $1 / 16$ & $1 / 256$ & $1 / 8$ & $1 / 128$ & $1 / 64$ & $1 / 64$ \\
\hline $\mathrm{N}^{\circ} 2$ & $1 / 4$ & $1 / 2$ & $1 / 128$ & $1 / 16$ & $1 / 128$ & $1 / 128$ & $1 / 256$ \\
\hline$N^{\circ} 3$ & $1 / 4$ & $1 / 8$ & $1 / 128$ & $1 / 8$ & $1 / 64$ & $1 / 64$ & $1 / 128$ \\
\hline
\end{tabular}

The AMP activity present in the different plasma samples was measured by bacterial growth inhibition assay with E. coli SBS363 as bacterial target. Numbers indicate the minimal dilution of the samples that allowed normal bacterial growth. Three independent experiments were performed. U.C.: unchallenged, PBS: PBSchallenged, E. coli: Escherichia coli CIP7624, X. nematophila: Xenorhabdus nematophila F1, X. mauleonii: Xenorhabdus mauleonii $\mathrm{VCO}^{\top}$. 
Table 4: Effect of supernatant from Xenorhabdus nematophila F1 culture on the expression of Spodoptera frugiperda antimicrobial peptides.

\begin{tabular}{lccccc} 
& \multicolumn{5}{c}{ Larval treatment } \\
\cline { 2 - 6 } Experiments & U.C. & PBS & E. coli & Live $X . n$. & $\begin{array}{c}\text { E. coli/ } \\
\text { Sup }^{(\text {(a) }} \boldsymbol{X} . \boldsymbol{n} \text {. }\end{array}$ \\
\hline $\mathrm{N}^{\circ} 1$ & $1 / 1$ & $1 / 16$ & $1 / 128$ & $1 / 16$ & $1 / 4$ \\
$\mathrm{~N}^{\circ} 2$ & $1 / 2$ & $1 / 4$ & $1 / 64$ & $1 / 4$ & $1 / 16$ \\
$\mathrm{~N}^{\circ} 3$ & $1 / 2$ & $1 / 16$ & $1 / 128$ & $1 / 16$ & $1 / 16$ \\
\hline
\end{tabular}

The AMP activity present in the different plasma samples was measured by bacterial growth inhibition assay with E. coli SBS363 as bacterial target. Numbers indicate the minimal dilution of the samples that allowed normal bacterial growth. Three independent experiments were performed. U.C.: unchallenged, PBS: PBS-challenged, E. coli: Escherichia coli CIP7624, X. n.: Xenorhabdus nematophila F1. ${ }^{(a)}$ Sup: Culture supernatant of $X$. nematophila was obtained after centrifugation and filtration $(0.22 \mu \mathrm{m})$ of an overnight bacterial culture. $20 \mu \mathrm{L}$ of $X$. nematophila culture supernatant was injected the same time than $E$. coli. 


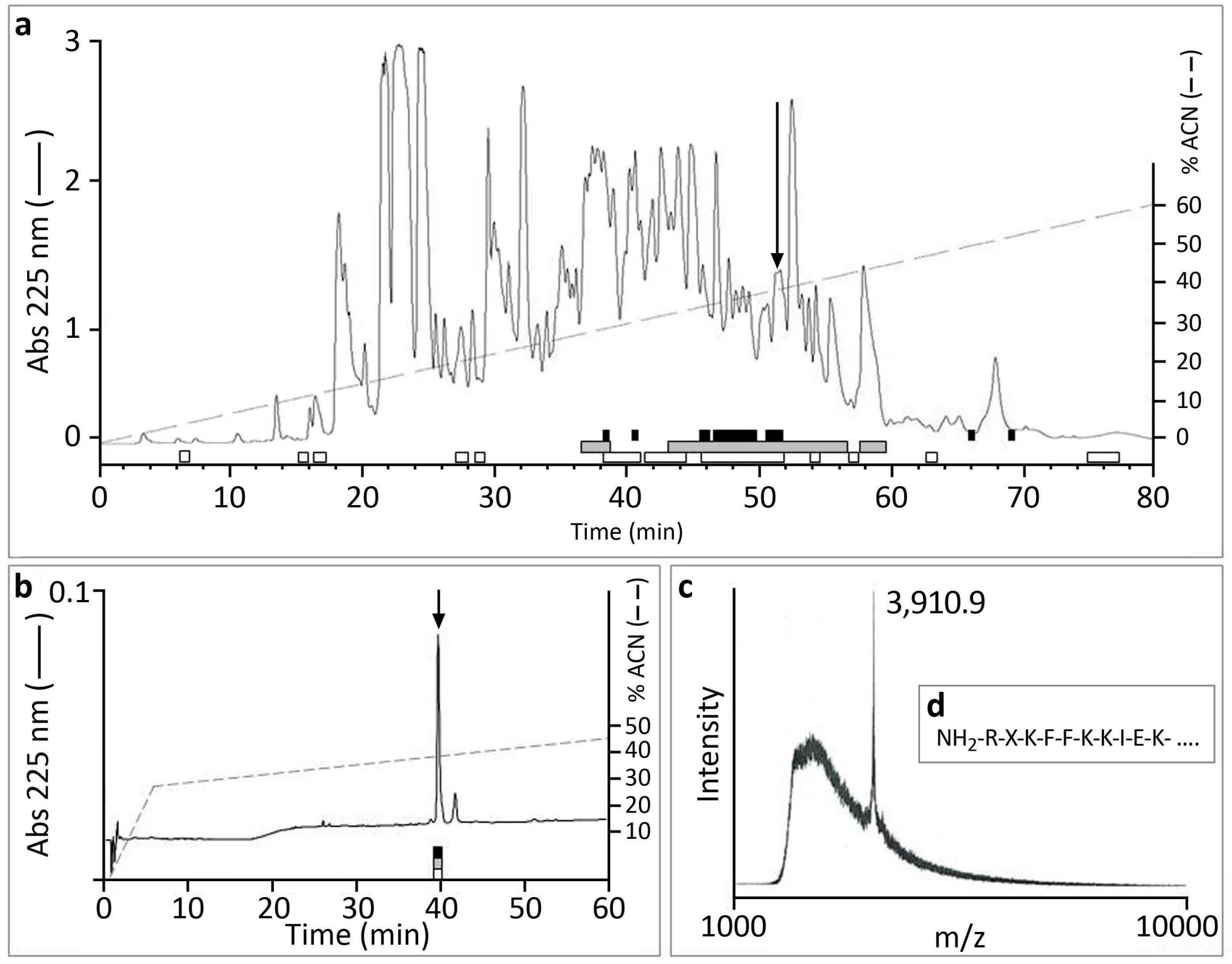


Figure 2

政

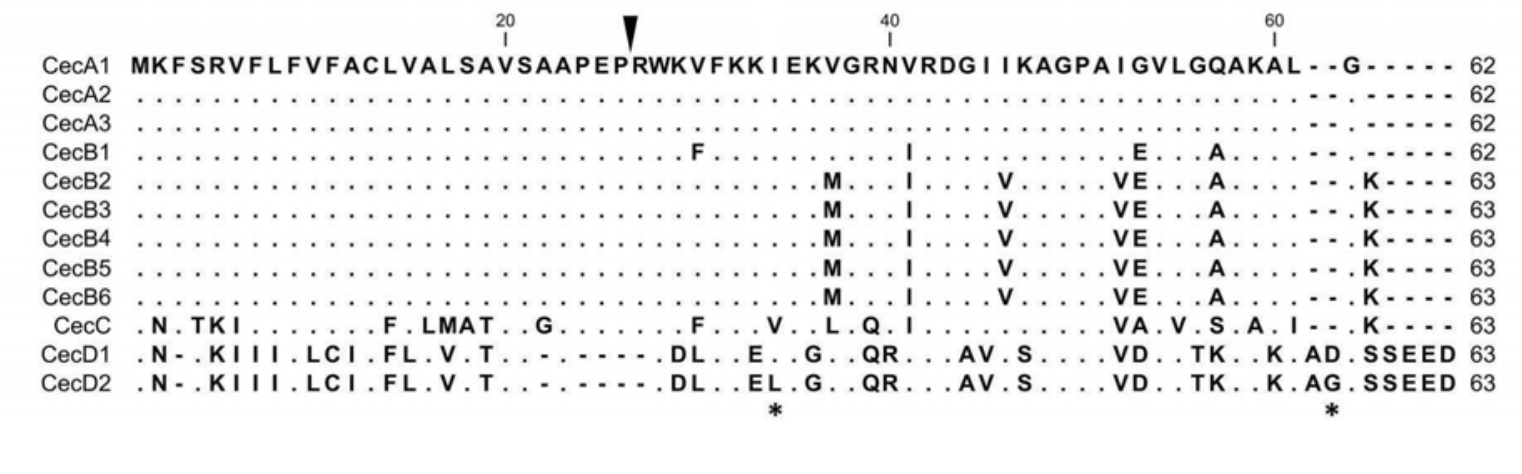

\section{0}

.

.

政
.

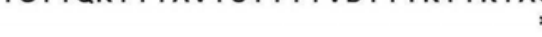

*

\section{Fure 2}




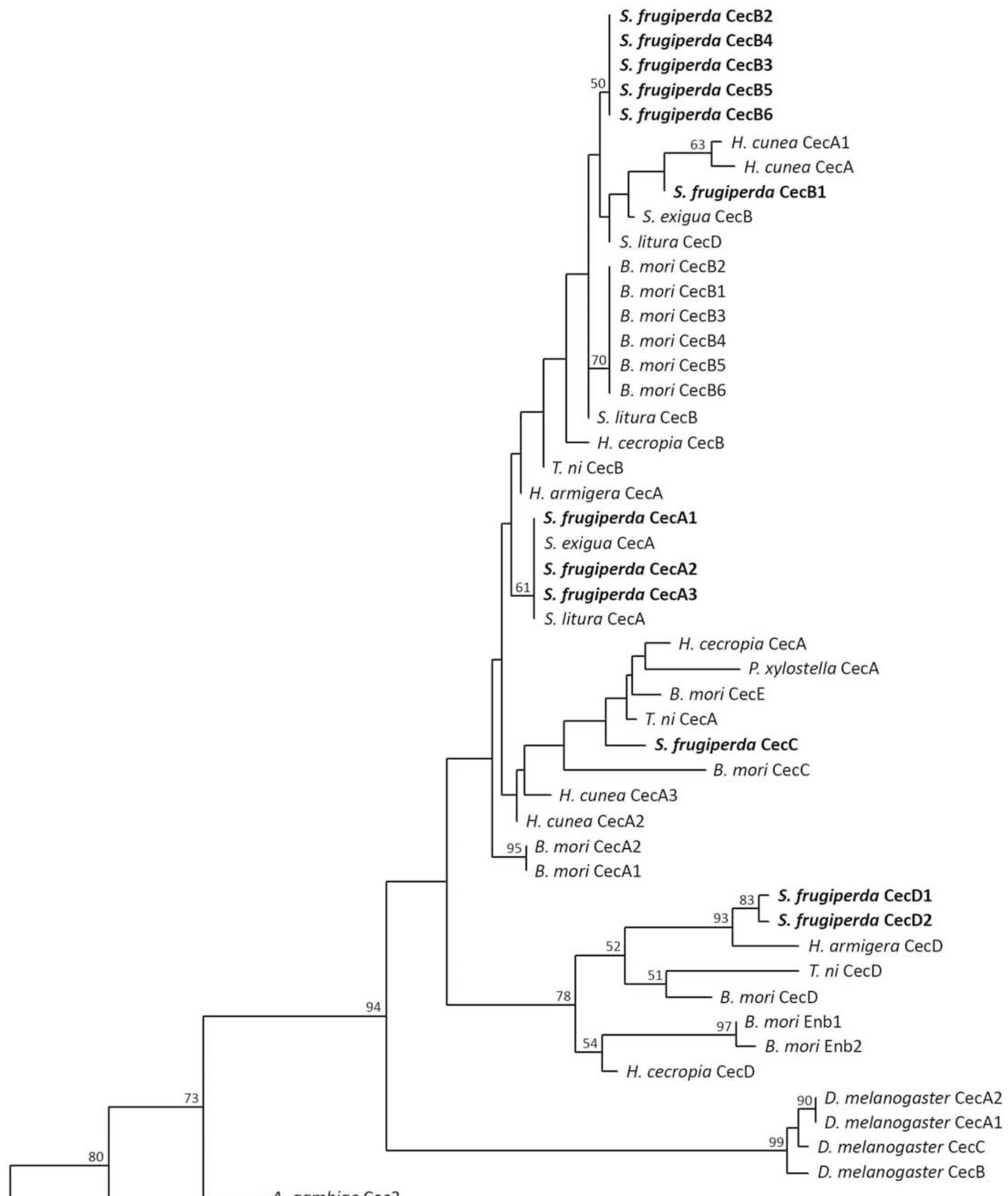

A. gambiae $\mathrm{Cec} 3$

A. gambiae Cec4

A. gambiae $\mathrm{Cec} 2$

A. gambiae Cec1 


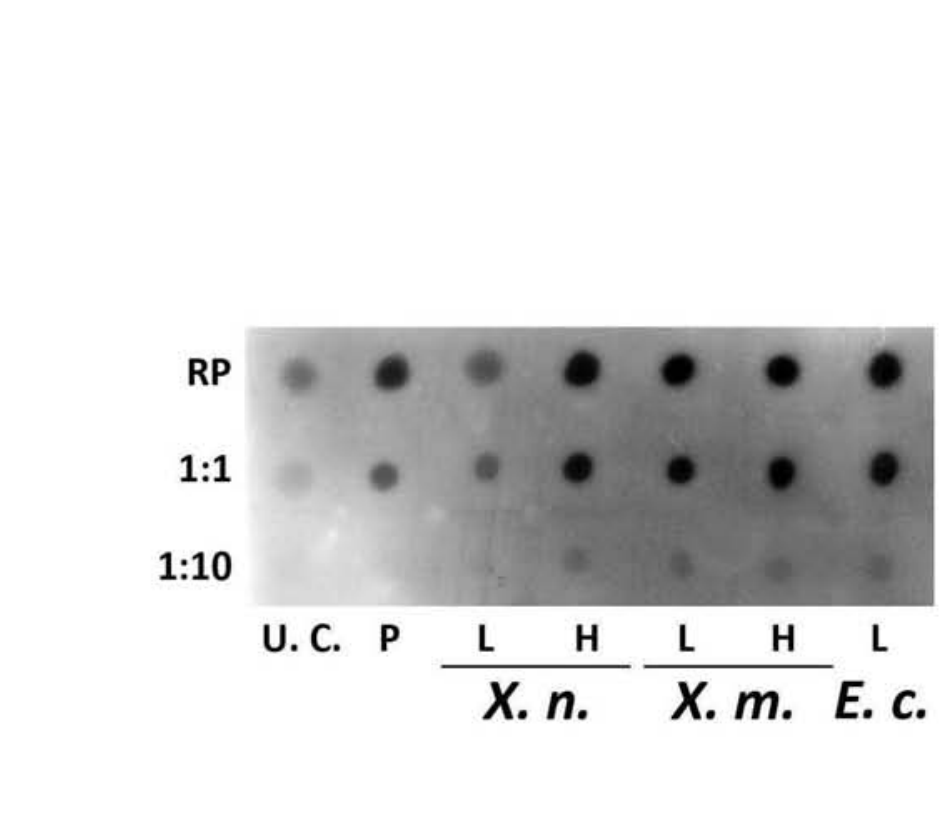

Figure 4

Figure 4

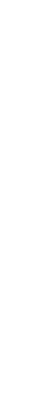


Supplementary Table 1: Sequences of insect cecropins used in this study.

\begin{tabular}{|c|c|c|}
\hline Species & Sequences & Acc numbers \\
\hline A. gambiae Cec1 & GRLKKLGKKIEGAGKRVFKAAEKALPVVAGVKALG & $\underline{X P \quad 311223}$ \\
\hline A. gambiae Cec2 & FKKFLKKVEGAGRRVANAAQKGLPLAAGVKGLVG & $\underline{X P \quad 311222}$ \\
\hline A. gambiae Cec3 & RWKFGKRLEKLGRNVFRAAKKALPVIAGYKALGA & $\underline{X P \quad 311224}$ \\
\hline A. gambiae Cec4 & LKKFGKKLEKVGKNVFHAVEKVVPVLQGIQDLRDKKNGQRG & $\underline{X P \quad 565481}$ \\
\hline B. mori CecA1 & RWKLEKKIEKVGRNVRDGLIKAGPAIAVIGQAKSLGK & ref. [31] \\
\hline B. mori CecA2 & RWKLEKKIEKVGRNVRDGLIKAGPAIAVIGQAKSLGK & ref. [31] \\
\hline B. mori $\mathrm{CecB} 1$ & RWKIEKKIEKMGRNIRDGIVKAGPAIEVLGSAKAIGK & ref. [31] \\
\hline B. mori CecB2 & RWKIFKKIEKMGRNIRDGIVKAGPAIEVLGSAKAIGK & ref. [31] \\
\hline B. mori $\mathrm{CecB} 3$ & RWKIFKKIEKMGRNIRDGIVKAGPAIEVLGSAKAIGK & ref. [31] \\
\hline B. mori CecB4 & RWKIFKKIEKMGRNIRDGIVKAGPAIEVLGSAKAIGK & ref. [31] \\
\hline B. mori CecB5 & RWKIFKKIEKMGRNIRDGIVKAGPAIEVLGSAKAIGK & ref. [31] \\
\hline B. mori CecB6 & RWKIFKKIEKMGRNIRDGIVKAGPAIEVLGSAKAIGK & ref. [31] \\
\hline B. mori CecC & KRKVFKIIEKIGRNVRGGVITAGPAVVVVGQAASVGM & ref. [31] \\
\hline B. mori CecD & GNFFKDLEKMGQRVRDAVISAAPAVDTLAKAKALGQG & ref. [31] \\
\hline B. mori CecE & RWKIFKKIEKVGQNIRDGIIKAGPAVAVVGQAATIAHGK & $\underline{A B B 19289}$ \\
\hline B. mori Enbocin1 & WNFFKEIERAVARTRDAVISAGPAVATVAAASAVASG & $\underline{\mathrm{AAC} 02238}$ \\
\hline B. mori Enbocin2 & WNFFKEIERAVARTRDAVISAGPAVATVGAAAAVASG & $\underline{\text { BAF51563 }}$ \\
\hline D. melanogaster CecA1 & GWLKKIGKKIERVGQHTRDATIQGLGIAQQAANVAATARG & NP 524588 \\
\hline D. melanogaster CecA2 & GWLKKIGKKIERVGQHTRDATIQGLGIAQQAANVAATARG & NP 524589 \\
\hline D. melanogaster $\mathrm{CecB}$ & GWLRKLGKKIERIGQHTRDASIQVLGIAQQAANVAATARG & $\overline{N P 524590}$ \\
\hline D. melanogaster $\mathrm{CecC}$ & GWLKKLGKRIERIGQHTRDATIQGLGIAQQAANVAATARG & NP 524591 \\
\hline H. armigera $\mathrm{Cec} \mathrm{A}$ & RWKVFKKIEKVGRNVRDGVIKAGPAIAVLGEAKALG & $\underline{\mathrm{AAX} 51304}$ \\
\hline H. armigera $\mathrm{CecD}$ & WDFFKELEGAGQRVRDAIISAGPAVDVLTKAKGLYDSSEEKD & $\underline{\text { AAX51193 }}$ \\
\hline H. cecropia CecA & KWKLFKKIEKVGQNIRDGI IKAGPAVAVVGQATQIAKG & $\underline{\text { CAA29871 }}$ \\
\hline H. cecropia $\mathrm{CecB}$ & KWKVFKKIEKMGRNIRNGIVKAGPAIAVLGEAKALG & $\underline{\text { AAA29187 }}$ \\
\hline H. cecropia CecD & WNPFKELEKVGQRVRDAVISAGPAVATVAQATALAKGK & $\underline{\text { AAX51193 }}$ \\
\hline H. cunea CecA & RWKIFKKIERVGQNVRDGIIKAGPAIQVLGTAKALGK & $\underline{\text { P50720 }}$ \\
\hline H. cunea CecA1 & RWKFFKKIERVGQNVRDGLIKAGPAIQVLGAAKALGK & $\underline{\text { P50721 }}$ \\
\hline H. cunea CecA2 & RWKVFKKIEKVGRNIRDGVIKAGPAIAVVGQAKALGK & $\underline{\text { P50722 }}$ \\
\hline H. cunea CecA3 & RWKVFKKIEKVGRHIRDGVIKAGPAITVVGQATALGK & $\underline{\text { P50723 }}$ \\
\hline P. xylostella CecA & RWKPFKKLEKVGRNIRNGI IRYNGPAVAVIGQATS IARPTGK & $\underline{\mathrm{ACX} 31606}$ \\
\hline S. exigua $\mathrm{Cec} A$ & RWKVFKKIEKVGRNVRDGI IKAGPAIGVLGQAKAL & ref. [20] \\
\hline S. exigua $\mathrm{CecB}$ & RWKVEKKIEKVGRNIDGI IKAGPAVEVLGTAKAL & ref. [20] \\
\hline S. frugiperda CecA1 & RWKVFKKIEKVGRNVRDGIIKAGPAIGVLGQAKALG & This study \\
\hline S. frugiperda CecA2 & RWKVFKKIEKVGRNVRDGIIKAGPAIGVLGQAKALG & This study \\
\hline S. frugiperda CecA3 & RWKVFKKIEKVGRNVRDGI IKAGPAIGVLGQAKALG & This study \\
\hline S. frugiperda $\mathrm{CecB} 1$ & RWKFFKKIEKVGRNIRDGIIKAGPAIEVLGAAKALG & This study \\
\hline S. frugiperda $\mathrm{CecB} 2$ & RWKVFKKIEKMGRNIRDGIVKAGPAVEVLGAAKALGK & This study \\
\hline S. frugiperda CecB3 & RWKVFKKIEKMGRNIRDGIVKAGPAVEVLGAAKALGK & This study \\
\hline S. frugiperda $\mathrm{CecB} 4$ & RWKVFKKIEKMGRNIRDGIVKAGPAVEVLGAAKALGK & This study \\
\hline S. frugiperda CecB5 & RWKVEKKIEKMGRNIRDGIVKAGPAVEVLGAAKALGK & This study \\
\hline S. frugiperda CecB6 & RWKVFKKIEKMGRNIRDGIVKAGPAVEVLGAAKALGK & This study \\
\hline S. frugiperda CecC & RWKFFKKVEKLGQNIRDGIIKAGPAVAVVGSAAAIGK & This study \\
\hline S. frugiperda CecD1 & WDLFKEIEGVGQRVRDAVISAGPAVDVLTKAKKLADGSSEED & This study \\
\hline S. frugiperda CecD2 & WDLFKELEGVGQRVRDAVISAGPAVDVLTKAKKLAGGSSEED & This study \\
\hline S. litura $\mathrm{Cec} A$ & RWKVFKKIEKVGRNVRDGIIKAGPAIGVLGQAKALG & $\underline{\text { Q9XZG9 }}$ \\
\hline S. litura $\mathrm{CecB}$ & RWKVFKKIEKMGRNIRDGIVKAGPAIEVLGSAKALGK & $\underline{\mathrm{Q} 9 X Z \mathrm{HO}}$ \\
\hline S. litura CecD & RWKVFKKIEKMGRNIRDGIIKAGPAVEVLGSAKALGK & $\underline{A B Q 51092}$ \\
\hline T. ni CecA & RWKFFKKIEKVGQNIRDGIIKAGPAVAVVGQAASITGK & $\underline{\mathrm{P} 50724}$ \\
\hline T. ni CecB & RWKVFKKIEKMGRNVRDGIIKAGPAIAVLGEAKALGK & $\underline{A B V 68872}$ \\
\hline T. ni CecD & GNFFKDLEGIGQRVRDAIESAGPAVDVLGRAAALSRGEQQQRE & $\underline{A B V 68873}$ \\
\hline
\end{tabular}


Legend of Supplementary Figures 2 to 6: Cecropin-encoding contigs (see Table 1) present in the Spodobase (Negre et al., 2006) were aligned using Clustal W. Identical nucleotides in Clusters I, II and V are boxed. In the case of Cluster III, boxes indicate residues which are identical in, at least, 3 sequences. ORFs are indicated by the black line.

Supplementary Fig. 2. Supplementary material.

\section{Cluster I}

S11F00836... AT TCGAAGACGAGCGCTTCTAGTAGCAAGATAGTAAATATTT T 9 AAAG TAAAGCAAAATAATT GTGAAGATGAAGT TC TCCCGAG TGT TCC TGT TC 96 S11F01169... ATTCGAAGACGAGCGCTTCTAGTAGCAAGATAGTAAATATTTTAAAAGTAAAACAAAATATTAGTAAAGATGAAGTTCTCCCGAGTGTTCCTGTTC 96 S11F04795 ATCATTCGAAGACGAGCGCTTCTAGTAGCAAGATAGTAAATATTTTAAAAGTAAAACAAAATAATAGTGAAGATGAAGTTCTCCCGAGTGTTCCTGTTC 99

$$
100
$$

120

140

160

Sf1F00836 GTGTTCGCGTGCCTGGTCGCGCTGAGCGCCGTCAGCGCCGCGCCAGAGCCGAGGTGGAAGGTCTTCAAGAAGAT TGAGAAAGTGGGCCGCAACGTAAGA 195 S11F01169 GTGTTCGCGTGCCTGGTCGCGCTGAGCGCCGTCAGCGCCGCGCCAGAGCCGAGGTGGAAGGTCTTCAAGAAGATTGAGAAAGTGGGCCGCAACGTAAGA 195 S1F04795 GTGTTCGCGTGCCTGGTCGCGCTGAGCGCCGTCAGCGCCGCGCCAGAGCCGAGGTGGAAGGTCTTCAAGAAGATTGAGAAAGTGGGCCGCAACGTAAGA 198

202002900290

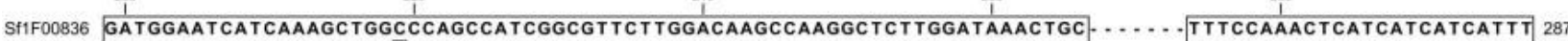
Sf1F01169 GATGGAATCATCAAAGCTGGACCAGCCATCGGCGTTCTTGGACAAGCCAAGGCTCTTGGATAAACTGC....... TTTCCAAACTCATCATCATCATTT 287 SH1F04795 GATGGAATCATCAAAGCTGGCCCAGCCATCGGCGTTCTTGGACAAGCCAAGGCTCTTGGATAAACAAACCAAACATCTACAAACTCATCATCATCATT TI 297

300

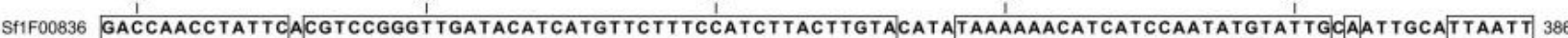

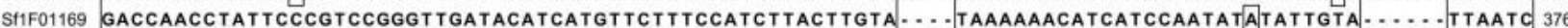
S11F04795 GACCAACCTET TCCCGTCCGGGT TGATACATCATGTTCTTTCCATCTTACTTGTA.... TTAAAG CATCATCCGATATGTAT TGTAT ..... TTAATC 387 400 420 400 460 480

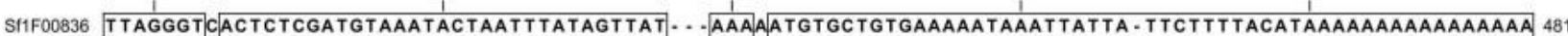

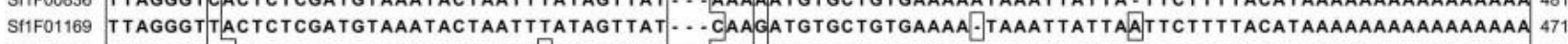

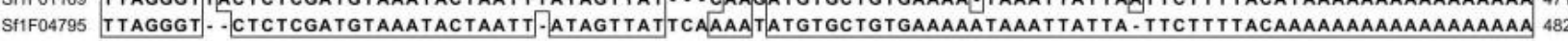
500

Sf1F00836 AAAAAAAAAAAAAAAAA..... 498

Sf1F01169 AAAAAAAAAAAAAAAAAAAAAAA 49

Sf1F04795 AAAAAAAAAAAAAAAAAAAAAAA 505

Supplementary Fig. 3. Supplementary material.

\section{Cluster II}

20

Sf1F02507 AGCATGGTATCAACGCAGAGTGCCCATTACGCCGgGGATCATTCGTTGTGTATCAACTGCAGAGCAATTT TGT TAT TAT TAAATAGCAT TATTATT TCA 99 SH1F09170 ..............................................

$$
100
$$

120

140

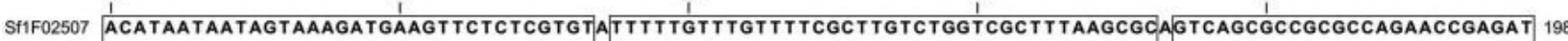
Sf1F09170 ACATAATAATAGTAAAGATGAAGTTCTCTCGTGTGTTTTGTTTGTTTTCGCTTGTCTGGTCGCTTTAAGCGCTGTCAGCGCCGCGCCAGAACCGAGAT 156

$$
200
$$

220

240

260

200

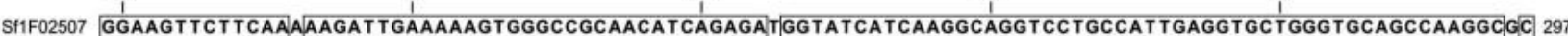
Sf1F09170 GGAAGT TCTTCAAGAAGAT TGAAAAAGTGGGCCGCAACATCAGAGACGGTATCATCAAGGCAGGTCCTGCCATTGAGGTGCTGGGTGCAGCCAAGGCAC 255$$
300
$$

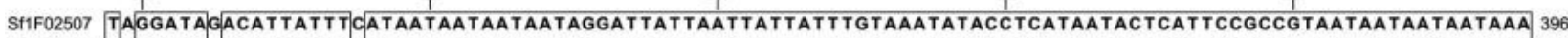
Sf1F09170 TGGGATAAACATTATTTIATAATAATAATAATAGGATTATTAATTATTATTTGTAAATATACCTCATAATACTCATTCCGCCGTAATAATAATAATAAA 354

$$
400
$$

$$
420
$$

$$
440
$$

$$
460
$$

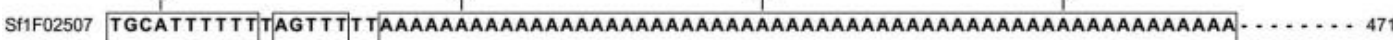
S11F09170 TGCATTTTTT.-AGTTTGGAAAAAAAAAAAAAAAAAAAAAAAAAAAAAAAAAAAAAAAAAAAAAAAAAAAAAAAAAAAAAAAAA 436 


\section{Supplementary Fig. 4. Supplementary material.}

\section{Cluster III}

S11F02553-2 ATCATTGAGAGCAGAGCCACAGAGTCGCGCAGAACAACACCAGTGCCACACAGCATCAGTA $\frac{1}{1}$. SHF02902 ATCATTGAGTGCAGAGCCACAGAGTCGCGCAGTACCGGCACCAGTGCCACACAGCATCAGTA ............................ S11F02563-1 ACCATTGAGTGCAGAGCCATAGAGTCGCGCAGTACATICACCAGTGCCACACAGCATCAGTACAGCTCCAGTGCCACACAGCATCAGTGCAGCATCAGGA 90

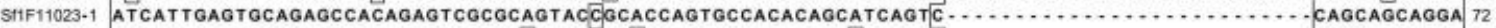

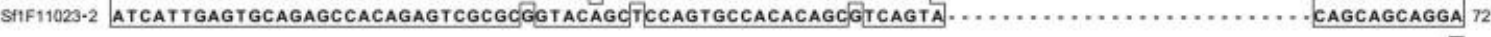

\section{0} 300 190

S1F02553-2 TGAAGTTCTCCCGAGTGTTCCTOTTCGTOTTCGCOTGCCTGGTCGCGCTGAGCGCCGTCAGCOCCGCGCCAGAGCCGAGGTGGAAGGTCTTCAAGAAGA 17 S1F02902 TGAAGTTCTCCCGAGTATTCCTGTTCGTGTTCGCGTGCCTGGTCGCGITGAGCGCCGTCAGCGCCGCGCCAGAGCCGAGGTGGAAGGTCTTCAAGAAGA 17 \$1F02553.1 TGAAGTTCTCCCGAGTGTTCCTGTTCGTGTTCGCGTGCCTGGTCGCGCTGAGCGCCGTCAGCGCCGCGCCAGAGCCGAGGTGGAAGGTCTTCAAGAAGA 198 S1F11023-2 TGAAGTTCTCCCGAGTGTTCCTGTTCGTGTTCGCGTGCCTGGTCGCGC TGAGCGCCGTCAGCGCCGCGCCAGAGCCGAGGTGGAAGGTCTTCAAGAAGA 171 $\begin{array}{llll}2000 & 200 & 1 & 1 \\ 1 & 1 & 1 & 1\end{array}$

S1F02563-2 TTGAGAAGATGGGCCGCAACATCAGAGACGG TATCG TCAAGGCAGG TCC TGC TGT CGAGG TG TTGGGTGCAGCCAAGGCGC TGGGGAAG TAATCAGCAG 270 S11F02902 TTGAGAAGATGGGCCGCAACATCAGAGACGGTATCGTCAAGGCAGGTCCTGCTGTCGAGGTGTTGGGTGCAGCCAAGGCGCTGGGGAAGTAATCAGCAG 270 S11F02553-1 TTGAGAAGATGGGCCGCAACATCAGAGACGGTATCGTCAAGGCAGGTCCTGCTGTCGAGGTGTTGGGTGAGCCAAGGCACTGGGGAAGTAATCAGCAG 297

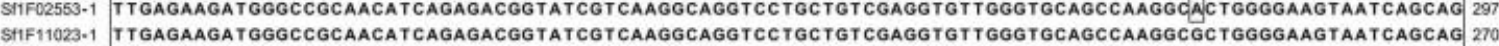

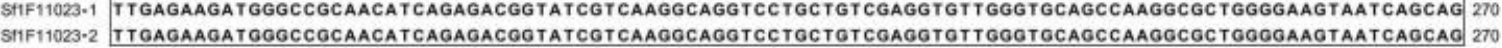

$$
300
$$

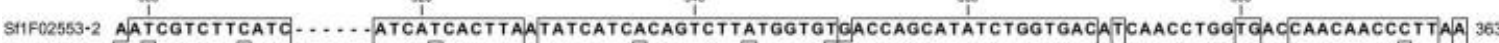

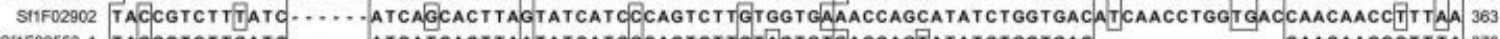

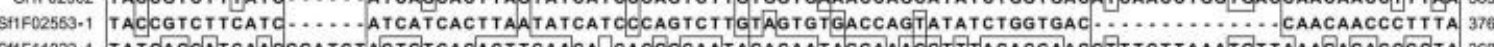

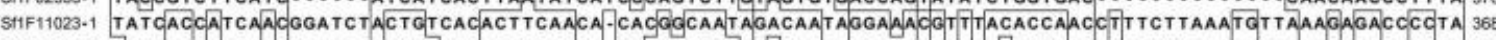

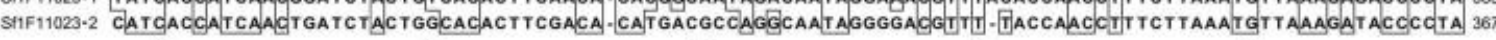

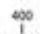
20 40.

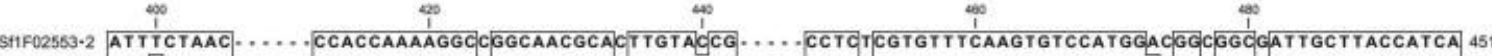

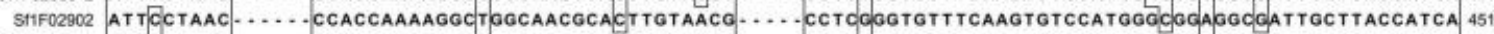

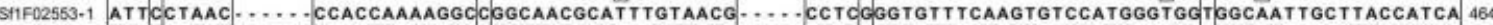

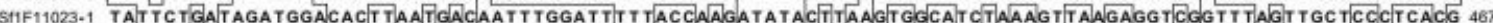

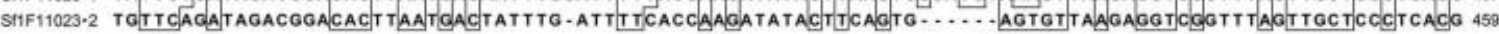

$$
100
$$
1 1 seo

S11F02553-2 GAAG

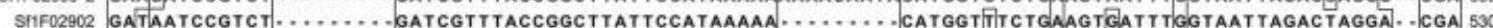

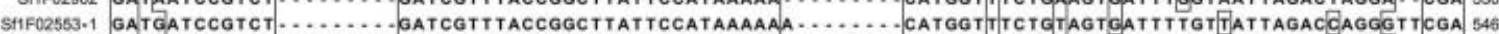

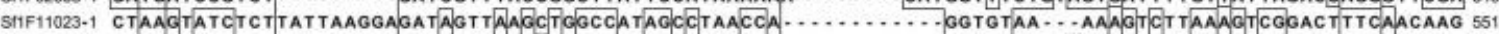

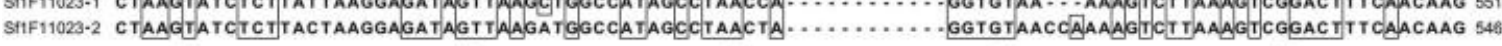<smiles>[LiH]</smiles>

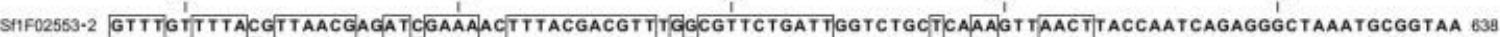

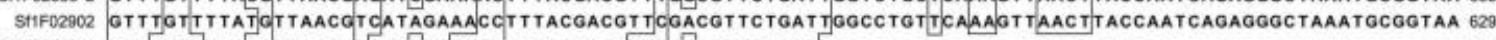

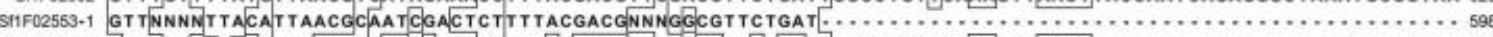

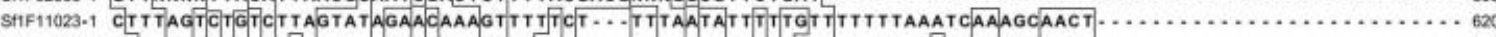

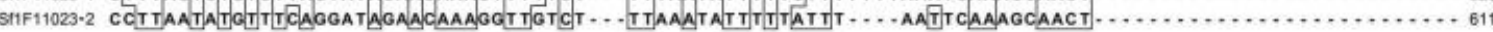

$$
\text { rom }
$$
Tro $\frac{740}{1}$ 760 760

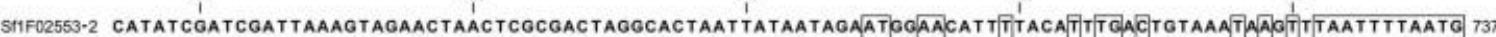
SHF 02902 CATATCGATCGATTAAAGTAGAACTAACTCGCAAATAGGCATTCTTAATAATAGAATGGAACAT TITACATT TAGCTGTAAATAAGT TTAATTITAATG 720 \$HF02553-1 . SHF11023-1

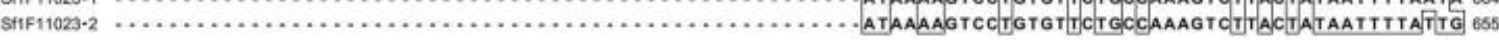

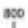
B⿺辶一 1 mo

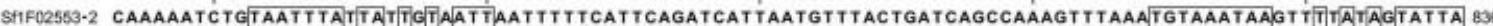

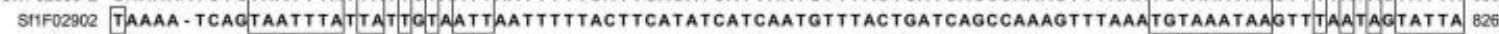

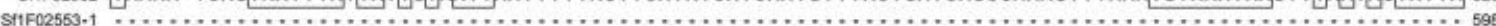

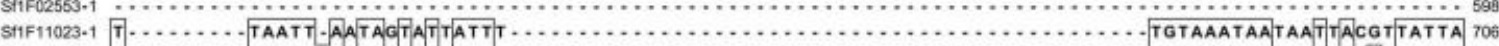

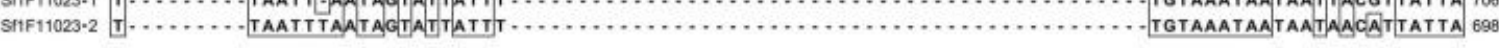
900 900

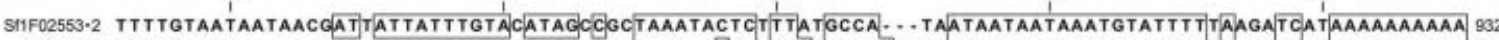

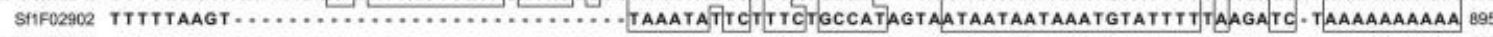
(1)............. 59

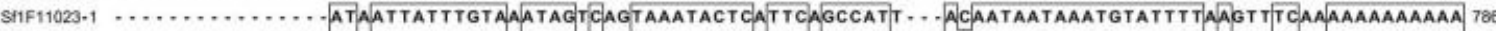

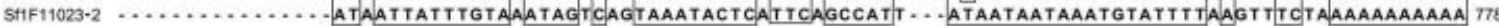
1000 100 100 1000

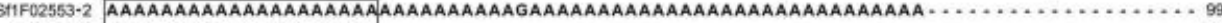

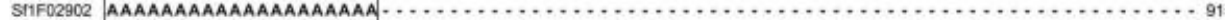

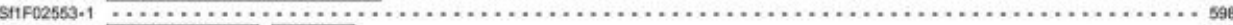

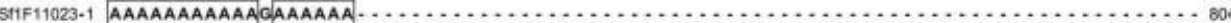

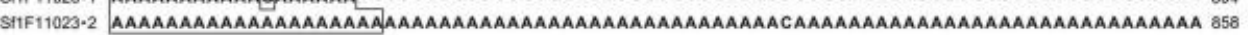


Supplementary Fig. 5. Supplementary material.

\section{Cluster IV}

Sf1F03731 ATCATHCGTGTCGTATCACTAGAGTTCGAAATAGAAAAATAATAATACATTTATTATTTTGCCATAATTAATAATTAATAA 100

120

160

SHF03731 ATAATAATAATGAATTTCACAAAGATATTTTTGTTTGTATTCGCTTGTTTTGTTTTGATGGCGACCGTGTCAGGTGCTCCTGAACCAAGGTGGAAATTC

2000220012000

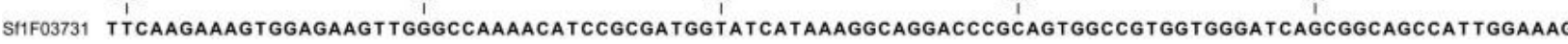

$3000390 \quad 300$

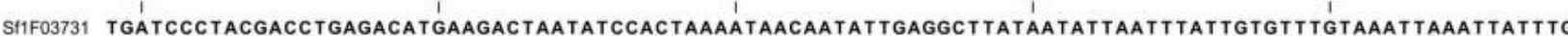

$$
400
$$

420

Sf1F03731 TAAGATAAAAAAAAAAAAAAAAAAAAAAAAAAAAAAA

Supplementary Fig. 6. Supplementary material.

\section{Cluster V}

$$
40
$$

Sf1F01749 GGTACTTGTTCATAGATCAGTTTCATT TTGAGCTTTCTCCAAGTAACAAGG TGCACGGTAGAGG TAAAGCCAAGT TGCAAAAAAAGCA AAAATGAAT TC 99

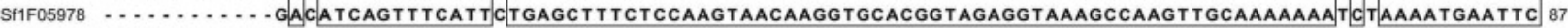

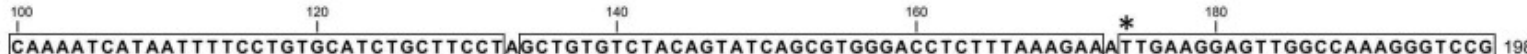
Sf1F05978 CAAAATCATAATTTTCCTGTGCATCTGCTTCCTGGCTGTGTCTACAGTATCAGCGTGGGACCTCTTTAAAGAACTTGAAGGAGT TGGCCAAAGGGTCCG 186 CAAAATCATAAT

$$
2002220 \quad 280020
$$

Sf1F01749 TGATGCTGTCATCAGTGCAGGACC TGCAGTAGACGTACTAACTAAGGCTAAAAAGC TGGC TGATGGATCCAGCGAAGAAGACTAGAAGACCATCA- GGT 296 Sf1F05978 TGATGCTGTCATCAGTGCAGGACCAGCAGTGGATGTGCTAACTAAAGCTAAAAAGCTGGCTGGAGGATCTAGCGAAGAGGACTAGAAACCCATCATGGT 285
30
300
320
360
380

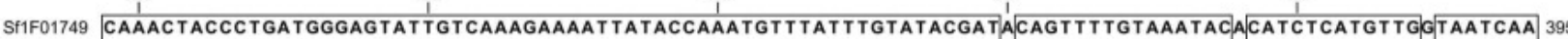
S11F05978 CAAACTACCCTGATGGGAGTATTGTCAAAGAAAATTATACCAAATGTTTATTTGTATACGATCCAGTTTTGTAAATAC CCATCTCATGT TGTTAATCAA 384

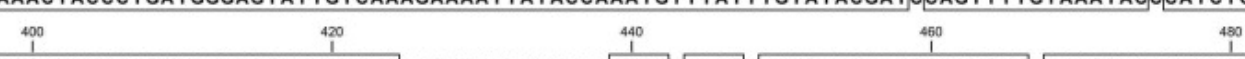

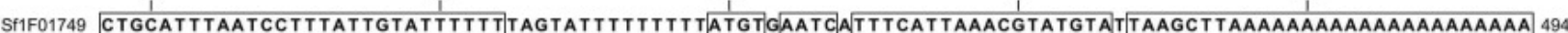

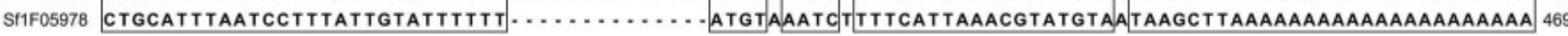

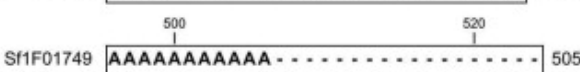

Sf1F05978 AAAAAAAAAAAAAAAAAAAAAAAAAAAAA 498 